Francisco Javier Ramírez Miranda

Noveno semestre, Licenciatura en Filosofía 


\section{Epistemología del testimonio ${ }^{1}$}

It seems, rather, that believing in other persons, in authority and testimony, is an essential part of the act of communicating, an act which we all constantly perform. It is as much an irreducible part of our experience as, say, giving promises, or playing competitive games, or even sensing coloured patches.

J.L. Austin, Other Minds

\section{Introducción}

Nuestra amplia dependencia del testimonio es evidente. Confiamos en el reporte de otros sobre una gran variedad de cosas, desde cuestiones concernientes a nuestro nacimiento - e.g., que nacimos tal día a tal hora, o que nuestra madre, de hecho, es nuestra madre - hasta descubrimientos en ciencia -e.g., que la tierra gira alrededor del sol, o incluso cualquier tipo de información geográfica-. Si careciéramos de él, nuestras vidas se verían fuertemente empobrecidas. Dada esta importancia, su estudio se ha incrementado de manera considerable. Actualmente, en la epistemología contemporánea, existen tres respuestas al problema de la justificación testimonial. La primera, que responde a la tradición, es una posición reduccionista, que afirma que el testimonio se «reduce» a la percepción, a la memoria o a las inferencias inductivas. La segunda es el denominado no reduccionismo, el cual considera que el testimonio es una fuente independiente de conocimiento y que nos encontramos prima facie justificados a menos que obtengamos una creencia o evidencia relevante en contra. La tercera y última es más reciente, desarrollada principalmente por Jennifer Lackey, pretende eliminar la dicotomía entre reduccionismo y no reduccionismo, posicionándose en medio de ambas posturas y rechazando, por un lado, «el componente reductivo» del reduccionismo y, por otro lado, la supuesta credulidad o «actitud acrítica» del no reduccionismo.

1 El presente ensayo forma parte de una investigación mucho más amplia respecto al problema del testimonio, la cual fue desarrollada con el apoyo de la AMC (Academia Mexicana de las Ciencias), durante el periodo del 29 de junio al 21 de agosto de 2015. Agradezco a Mario Gensollen Mendoza, quien ha sido un gran maestro. Los últimos dos años de trabajo con él han sido sumamente enriquecedores. No obstante, cabe hacer la aclaración que todo lo dicho aquí es responsabilidad mía. 
El presente ensayo es expositivo, comprende una parte de una investigación mucho más amplia. Pretendo dar un breve panorama del debate en torno a la epistemología del testimonio. No obstante, hago énfasis en la crítica que Jennifer Lackey ofrece contra el no reduccionismo. Creo que los contraejemplos que elabora no proceden. Por el contrario, el agente en cuestión cuenta con contra-creencias o contra-evidencia (más precisamente, un derrotero por su término en inglés: defeater) que le indica la fiabilidad o no fiabilidad del testimonio.

El orden que seguiré es el siguiente: en primer lugar, me concentro en las características generales del debate en torno al problema del testimonio; luego expongo la crítica a los contraejemplos ofrecidos por Lackey, argumentando que los agentes en cuestión cuentan con un derrotero normativo que les obliga a considerar al testimonio como no fiable. ${ }^{2}$

\section{Debate reduccionismo-no reduccionismo}

¿Cuándo se encuentra justificado un agente al aceptar el testimonio de un tercero? Esta pregunta es la preocupación principal de la epistemología del testimonio y ha ocupado a una amplia gama de filósofos que pretenden responderla. Como vimos, en la literatura contemporánea existen tres posiciones, que enseguida analizaremos brevemente.

\section{(a) Reduccionismo}

Según Lackey, existen dos componentes centrales del reduccionismo. El primero es «el componente de razones positivas», el segundo «el compo-

2 El testimonio que se discutirá en el presente artículo es el denominado "Testimonio Natural", del cual Coady dice: "[...]El testimonio natural se encuentra a diario en todos los contextos, que exhibe las operaciones sociales de la mente: darle a alguien alguna dirección, reportar qué ha pasado en una accidente, decir que sí, que has visto a un niño responder a esta descripción, decirle a alguien el resultado de la última carrera o la puntuación en el cricket" (Coady, C.A.J. Testimony: A Philosophical Study, Oxford, 1992, p. 38). Existe una segunda clase de Testimonio, el cual puede entenderse como “Testimonio Formal”, el cual puede encontrarse en los juzgados. Para una aproximación formal del testimonio véase, por ejemplo, Goldman, A., Knowledge in a Social World, Oxford, 1999, cap. 4. 
nente reductivo» ${ }^{3}$. El primero de ellos señala que el proceso de justificación consiste en la presencia de razones especiales, es decir, es necesario que exista un trabajo positivo por parte de un oyente $H$. En la medida que se pretende evitar la circularidad en el proceso justificatorio del testimonio, es decir, en donde las razones no dependan del testimonio mismo, su justificación se hace depender de otras fuentes distintas de conocimiento, i.e., de la memoria, de la percepción o de las inferencias inductivas. Aquí es donde nos topamos con el segundo componente, el componente reductivo, puesto que en la búsqueda de la no circularidad se responde con razones no testimoniales, reduciéndolo así a un proceso interno-individual.

No obstante, ambos componentes se presentan de dos maneras: o bien pueden aparecer trabajando en conjunto o sólo el componente de razones positivas. En el primer caso encontramos dos clases de reduccionismos, mientras que en el segundo al hibridismo de Lackey. Comencemos con las dos clases de reduccionismos: reduccionismo global y reduccionismo local.

El reduccionismo global es una versión débil y fácilmente derrotable. El argumento principal que el reduccionismo global ofrece es que el agente en cuestión debe tener razones positivas no testimoniales para creer que el testimonio es generalmente fiable. Es decir, el testimonio es una fuente derivada de conocimiento. Aunque nos encontremos justificados al confiar en el reporte de otros - nos dice el reduccionismo global- ello se debe a lo que nos han enseñado las fuentes no derivadas de conocimiento.

Existen problemas graves con esta posición. El primero de ellos lo ha señalado ya Thomas Reid, el clásico representante del no reduccionismo. En Essays on the Intellectual Powers of Man, Reid argumenta que si el reduccionismo (global) fuese cierto, entonces los niños serían incapaces de adquirir las herramientas necesarias para elaborar inferencias inductivas, las cuales son adquiridas por medio del testimonio de los padres. Una segunda razón para dudar de la veracidad del reduccionismo global, fue ofrecida por otro de los grandes no reduccionistas, C.A.J. Coady. En Testimony arguye que necesitaríamos estar expuestos a una amplia muestra de reportes y hechos correspondientes para inferir la fiabilidad gene-

3 Lackey, J., "It Takes Two to Tango: Beyond Reductionism and Non-Reductionism in the Epistemology of Testimony", en Lackey, J. y Sosa, E. (Ed.), The Epistemology of Testimony, Oxford, 2006, p. 160. 
ral. Pero la base observacional de los hombres comunes y corrientes es limitada, pues "muchos de nosotros nunca hemos visto un bebé nacer, ni muchos de nosotros hemos examinado la circulación de la sangre ni la geografía actual del mundo [...] ni un vasto número de otras observaciones que el reduccionismo parece requerir". $\mathrm{El}$ reduccionismo global, en vez de llevarnos a la credibilidad del testimonio, dada su impractibilidad, nos lleva inevitablemente al escepticismo.

Una tercera razón, y al parecer definitiva, cuestiona si incluso el reduccionismo global es un hecho epistémicamente significativo. ${ }^{5} \mathrm{El}$ argumento consiste en señalar la heterogeneidad que el testimonio presenta, dado su carácter contextual. Existen reportes de la hora del día, lo que hay para el desayuno, el carácter de los diferentes oponentes políticos, la edad y el peso de uno, el récord criminal, etcétera. Algunos de ellos son generalmente fiables - la hora del día y lo que hay para el desayuno-, otros menos fiables - el carácter de los diferentes oponentes políticosy otros relativos al hablante - la edad y el peso o el récord criminal-. Así, argumentar que el testimonio no es generalmente fiable no tiene importancia significativa en casos concretos.

El reduccionismo local, por su parte, es una versión refinada que es compatible con la heterogeneidad del testimonio. Este reduccionismo requiere que un oyente esté justificado en creer que un hablante particular $H$, cuyo testimonio actual es el punto de análisis, es fiable acerca de un tema en específico. El reduccionismo local, no obstante, continúa conservando una exigencia fuerte, pues argumenta que un oyente $S$ está justificado en el reporte particular de un hablante $H$ "si y sólo si" posee evidencia positiva o justificación. Es decir, considera que las razones positivas son necesarias y suficientes, por lo cual conserva el componente reductivo.

Varios contraejemplos se pueden elaborar al señalar que las razones positivas no son suficientes, pues a pesar de que ellas se cumplan y exista una supuesta reducción, dicho proceso no es practicable o no es un buen conductor a la verdad. Supóngase que María conoce a Pedro desde hace ya bastante tiempo, durante el cual ha obtenido buenas razones para creer en su fiabilidad: la información que ha obtenido de él siempre ha correspondido con un hecho concreto. En una ocasión, Pedro le dice a María que Ramón, un amigo en común, es una persona bastante

4 Coady, C.A.J., op. cit., p. 82.

5 Cfr., Lackey, J. op. cit., p. 162.

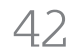


confiable, sobre todo cuando habla acerca de botánica. A causa de esto, María cree en el testimonio de Ramón con plena confianza de que las "flores esqueleto" se vuelven transparentes al contacto con el agua. A pesar de que Pedro es una persona fiable, se ha equivocado en esta ocasión. Ramón es un creyente incompetente y hablante nada sincero, sobre todo en cuestiones de botánica. Además, el proceso por medio del cual llegó a esa creencia fue una decisión azarosa; en alguna parte escuchó que algunas plantas adquirían transparencia cuando tenían contacto con el agua. Tras no recordar el nombre de las mismas abrió un libro de botánica y decidió que la primera flor que apareciese al hojearlo sería la poseedora de dicha característica.

Observemos que el presente caso pretende destacar que el hablante es un creyente nada competente y no fiable. Esto implica dos cuestiones: primero, que las razones positivas no son suficientes, porque María tenía razones objetivas y convincentes para creer en el testimonio de $\mathrm{Ra}$ món, es decir, el testimonio continuamente fiable y la sinceridad de $\mathrm{Pe}$ dro; segundo, se desaparta de un caso común de Gettier, puesto que la razón por la que las razones positivas no son suficientes no es a causa de la accidentalidad del conocimiento sino de las propiedades epistémicas del agente (su no fiabilidad y su incompetencia como creyente). Por tanto, el componente de las razones positivas, entendido en su sentido fuerte, es falso y, en consecuencia, el componente reductivo también.

\section{(b) No reduccionismo e hibridismo (dualismo)}

A diferencia del reduccionismo y del hibridismo - de los cuales el primero (ya sea local o global) considera que el componente de razones positivas es necesario y suficiente, y el segundo sólo que es necesario- el no reduccionismo afirma que el componente de razones positivas no es necesario ni suficiente. Más bien cree que se deben cumplir principalmente dos condiciones: (1) el reporte debe haber sido producido fiablemente, es decir, que un hablante $H$ sea un creyente competente y sincero; (2) un oyente $O$ no debe tener derroteros relevantes ${ }^{6}$ para tal reporte. Por tanto, en la medida que no exista evidencia en contra del testimonio, el oyente no tiene que hacer ningún trabajo epistémico positivo para la jus-

6 Existen dos tipos de derroteros: normativos y psicológicos. Los primeros son creencias o duda que son tenidas por un oyente $O$, mientras que los segundos son aquellas que deben tenerse - lo tenga o no el oyente-. 
tificación de su creencia testimonial. Permítaseme llamar al primero, por mera practicidad, "el componente de razones negativas" y al segundo «el componente no reduccionista". El componente de razones negativas tiene su origen en palabras de Tyle Burge, quien lo expresa de la siguiente manera: "Una persona tiene el derecho en aceptar como verdadero algo que es presentado como verdadero y es inteligible para ella, a menos de que existan razones fuertes para no hacerlo"? Por su parte, el componente no reduccionista evita que el proceso justificatorio testimonial se lleve a cabo únicamente dentro del cráneo de un oyente $O$, siendo además, de este modo, un posicionamiento claramente naturalista. Este segundo componente ha sido añadido por algunos no reduccionistas, debido a que se creía que la condición de un solo componente, el componente de razones negativas, eran muy débil. Del mismo modo, como podemos observar, el componente no reduccionista puede funcionar como parche en el contraejemplo de María y Pedro, en donde Ramón era un creyente nada competente y un hablante nada sincero. De esto último, Lackey es sumamente consciente, razón por la cual incluirá en su posición al componente no reduccionista, de modo que las dos condiciones de su dualismo sean las siguientes: (1) el reporte debe haber sido producido fiablemente, es decir, que un hablante $H$ sea un creyente competente y un sincero; (2) es necesario, mas no suficiente, que un oyente $O$ lleve a cabo un trabajo epistémico positivo, es decir, que ofrezca razones adecuadas para la justificación de su creencia testimonial. Finalmente, como podemos ver, la segunda condición de Lackey es incompatible con el componente no reduccionista, sin ser ella misma reduccionista.

Así, Jennifer Lackey ha elaborado un argumento que intenta rechazar al no reduccionismo. Y lo ha hecho a través de un contrajemplo que transcribo enseguida:

ALIEN: Sam, un ser humano común y corriente, toma una caminata por el bosque en un día soleado $y$, a la distancia, ve a alguien dejar un libro. Aunque la apariencia física del individuo le permite a Sam identificarlo como un alien de otro planeta, no sabe nada acerca de este tipo de alien o del planeta de donde proviene. Eventualmente, Sam pierde de vista al alien, pero es capaz de recuperar el libro que dejó. $\mathrm{Al}$ abrirlo, inmediatamente nota que parece estar escrito en inglés y luce como lo que llamamos en la tierra un diario. Además, tras leer la primera línea

7 Burge, T., “Content Preservation”, en The Philosophical Review, 102, 1993, p. 457. 
del libro, Sam forma la creencia correspondiente de que los tigres se han comido a algunos de los habitantes del planeta del autor. Resulta que el libro es un diario, el alien se comunica en inglés, y que se encuentra escrito tanto fiable como verdaderamente que los tigres se han comido a algunos de los habitantes del planeta en cuestión. ${ }^{8}$

Por otra parte, en la medida que Lackey pretende evitar la objeción de que el acto testimonial es una institución socialmente humana y, por tanto, que ALIEN no procede, introduce el siguiente ejemplo:

SALLY: Sally ha estado en coma por los dos meses pasados y, al caminar, descubre que ha perdido todo el conocimiento previo excepto su comprensión y manejo del inglés. Tras dejar el hospital, se topa con un diario de un autor desconocido y comienza a leerlo. Ahora, ex hipothesi, Sally no tienen más el sentido común de las creencias acerca de la psicología humana, no tiene más creencias acerca de la fiabilidad general de los humanos como testificadores, no tiene idea de cómo funcionan los diarios en nuestras sociedades, etcétera. ¿Sally está justificada en aceptar el contenido del diario?

Los ejemplos expuestos intentan señalar algo en particular: la falta de razones positivas por parte del agente. No posee, por ejemplo, la teoría psicológica de sentido común de los alien, no tiene creencias acerca de la fiabilidad general de los alien como testificadores, no posee creencias acerca de la fiabilidad del autor del libro, no tiene creencias sobre como los diarios funcionan en la sociedad del alien, etcétera. En la medida que el oyente carece de esto, y que el no reduccionismo, según Lackey, afirma que está justificado en creer el reporte del diario, éste cae una situación irracional, incluso mucho más que cuando se tiene contraevidencia sobre un testimonio (es decir, incurre en una "actitud acrítica", ingenua). Así, la creencia testimonial de Sam sólo estará justificada si posee razones positivas que descarten la posibilidad de que no, aún después de todo, habla inglés, de que su psicología folk es totalmente diferente a la nuestra o que sus diarios se parecen a los nuestros. ${ }^{10}$

8 Lackey, J., op. cit., p. 167.

9 Ibidem, p.169.

10 Cfr. Lackey, J., Learning from Words: Testimony as a Source of Knowledge, Oxford, 2008, pp. 169-173. 


\section{En defensa del no reduccionismo}

El argumento contra el reduccionismo puede reconstruirse de la siguiente manera:

(1) Si el no reduccionismo es verdadero, entonces en ALIEN Sam sabe que los tigres se han comido a algunos habitantes del planeta del autor del libro.

(2) Pero Sam no lo sabe (puesto que carece de razones positivas).

(3) Por tanto, el no reduccionismo, es falso.

Mi objetivo entonces es señalar que (1) es falso. Considero que Sam tiene un derrotero para no creer en el testimonio del alien. A esto Lackey respondería inmediatamente que no existe ningún derrotero que Sam pudiera tener, puesto que no sabe nada acerca de los alien (incluyendo su psicología y su comportamiento en sociedad). Precisemos, entonces.

Quiero comenzar con un argumento expuesto por Martin Kusch, el cual señala que ALIEN y SALLY trabajan con dos supuestos que no les permite refutar al reduccionismo. Los supuestos son los siguientes: la cualidad de persona y la presuposición no reduccionista de verdad. En la medida que se encuentren trabajando en conjunto, el argumento de Lackey no socava la presuposición no reduccionista de verdad. ${ }^{11}$ Veamos por qué.

Una objeción intuitiva que podría hacérsele a Lackey es que exige razones positivas para las creencias testimoniales y no para otra fuente distinta de conocimiento, por ejemplo, para la percepción. Y si son necesarias para su justificación, entonces enfrentaría el problema tradicional de la justificación epistémica, a saber: el regreso al infinito. La respuesta a este cuestionamiento resulta obvia: Lackey señala, correctamente, que las diferencias entre el testimonio y la percepción (o cualquier otro fuente interna de justificación), es que el primero presenta información intencional, mientras que el segundo no. Es decir, el testimonio es una institución social humana y, por tanto, debemos tener razones positivas a favor de su humanidad. Por lo tanto, en ALIEN y en SALLY se pone

11 Cfr. Kusch, M., "Jennifer Lackey on Non-Reductionism: A Critique”, 2011, pp. 4 -5. Recuperado de [https://www.academia.edu/1166002/Jennifer_Lackey_on_NonReductionism_A_Critique]. 
en entredicho que el agente tenga conocimiento de la cualidad de persona de quien profiere el testimonio.

Pero, si el supuesto de cualidad de persona se encuentra trabajando en conjunto con el de presuposición de verdad, entonces los contraejemplos fallan en aislar adecuadamente y, por tanto, refutar al no reduccionismo. En otras palabras, el no reduccionista está de acuerdo que un oyente $O$ necesita razones positivas para satisfacer la condición de cualidad de persona, pero no en que el agente tenga que ofrecer razones positivas a favor de la justificación del testimonio; dos casos, por supuesto, independientes. ${ }^{12}$ La crítica, por tanto, se encuentra parcialmente desviada.

Siendo esto así, pensemos en la gran cantidad de conocimiento de fondo, ${ }^{13}$ por medio del cual podemos aprender varias maneras en que el

12 Cfr. Ibidem, p. 5.

13 Adler ha enumerado la evidencia de fondo que poseemos para atribuir una confianza general al testimonio. La ha dividido en cinco puntos: "(1) El predominio de la verdad en el testimonio: abrumadoramente el testimonio transmite verdad (de manera no accidental). Ya que las acciones a menudo sobrevienen del testimonio nos encontramos en una posición adecuada para identificar su insuficiencia y ser responsables de la misma. Por tanto, es plausible concluir que el testimonio es ampliamente confiable. (2) La verdad como la norma: la verdad es una presuposición de la práctica de comunicación lingüística. La verdad gobierna el intercambio conversacional. [...] (3) Reputación y sanciones: la expectativa de verdad es suficientemente fuerte, de tal modo que aquellos que son víctimas de un testimonio falso, ya sea a través del error o la decepción, pueden llegar a confiar menos. Pero el alcance de esta pérdida de confianza será muy estrechamente circunscrita, en parte porque carecemos de otras formas diferentes al testimonio por medio de las cuales obtenemos información. En comunidades pequeñas y en marcos institucionales, aunque mucho menos en comunidades grandes donde el anonimato puede ser conservado, la reputación y las sanciones son un fuerte constreñimiento. Los columnistas que ofrezcan meros 'rumores' (chisme; Gossip) probablemente no conserven su trabajo, si los hechos verificados no coinciden con su testimonio. (4) El conocimiento impersonal de nuestros informantes: nuestros informantes (y nosotros) dependerán de sus creencias principalmente de fuentes confiables tales como la percepción, memoria, razonamiento y testimonio. El desarrollo moral estándar, la crianza y la educación tiene como propósito inculcar valores fundamentales, honestidad y preocupación por los otros, en particular. Estos valores son fácilmente enseñados por la obvia ventaja y beneficio mutuo de recibir información precisa. Nosotros aprendemos del tipo de hablantes quienes pueden ser menor o mayormente confiables, dependiendo de las circunstancias, así como 
testimonio fiable o no fiable. Esto apunta hacia nuestra continua y diaria exposición al mismo. Esto es: el estatus epistémico del testimonio es positivo. Asimismo, señalemos que la intención de ALIEN es no sólo mostrar nuestra inconforme intuición con la respuesta que pudiera dar el no reduccionismo, sino, antes de eso, la posibilidad de la hipótesis escéptica de que el agente, bajo los presupuestos del no reduccionismo, pueda saber si es un testimonio fiable o no. No obstante, creo que tomando en cuenta este conocimiento de fondo, es posible desmentir esta hipótesis escéptica; y esto a causa de la aparición de un derrotero y no por medio de una inferencia inductiva (o de un proceso interno). ${ }^{14}$ Es decir, bajo la aceptación de razones negativas y no positivas. ${ }^{15}$

En este sentido, Sam se ha topado con una novedosa e inusual fuente de conocimiento, el alien. Como lo sabemos, ignora todo sobre él, i.e. todo conocimiento de fondo que implique la elaboración de una razón positiva sobre su testimonio. Entonces, al parecer, no puede saber nada sobre su fiabilidad, pues no cuenta con evidencia alguna. No obstante, Sam sí cree que el diario fue dejado por un alien y que muy probablemente nunca antes ha interactuado con un individuo de su misma especie.

Según el no reduccionismo, si nos enfrentamos con una nueva fuente de conocimiento estaremos justificados prima facie. Pero esto, sólo en el caso de que tengamos contacto con seres humanos, puesto que sole-

de los temas en los que los extraños son más o menos competentes. (5) Motivación, relaciones socio-morales y cooperación: muy ocasionalmente mentir se encuentra bien motivado [...]. En general, sólo ocasionalmente los hablantes tienen mucho que ganar transmitiendo testimonio falso o injustificado" (Adler, J., "Epistemological Problems of Testimony", 2012. Recuperado de [http://plato.stanford.edu/entries/ testimony-episprob/].

14 Ciertamente, el argumento no es novedoso. Ha sido también expuesto recientemente por Timothy Perrine (ver, Perrine, T., "In Defense of Non- Reductionism in the Epistemology of Testimony”, Synthese, 191, 2014, pp. 3227-3237). La diferencia entre el presente ensayo y el de Perrine es que intento clarificar, a partir del argumento de Kusch, porque esto sucede, además de algunas cuestiones más que deben encontrarse dentro de la agenda de la epistemología del testimonio.

15 Aunque esto no quiere decir que el agente no sea capaz de ofrecer razones positivas a favor de su creencia. Lo importante, no obstante, es resaltar que no son prerrequisito para el conocimiento o justificación. Como lo señala Kornblith, pueden sobrevenir en el proceso justificatorio o simplemente no presentarse (Cfr. Kornblith, H. Knowledge and its Place in Nature, Oxford, cap. 3. También ver Kornblith, H.,"The Role of Reasons in Epistemology", Episteme, 12, 2015, pp. 225-239). 
mos compartir un conjunto de conocimiento de fondo sobre el testimonio. Sam no lo tiene (y está consciente de ello) porque presumiblemente el reporte lo ha escrito un alien y sabe que nunca antes ha interactuado con uno de su misma especie. Así, esto le generaría un derrotero no en razón de la fiabilidad del hablante, sino en que es una fuente nueva e inusual de conocimiento, por lo cual, según su conocimiento de fondo implícito, probablemente no lo lleve a la verdad. Además, esta contra-creencia que ha generado Sam es de tipo normativa, es decir, es una creencia que debe tener. Luego, la aparición de una contra-creencia no le permite adquirir a este testimonio el estatus de conocimiento; por tanto, siguiendo al no reduccionismo, Sam no se encuentra justificado.

Lackey podría argumentar que Sam no posee un derrotero normativo, puesto que no pose evidencia positiva alguna. Es decir, no podría creer que algunos de los alien no fueron tragados por los tigres o que el diario no es fiable. Pero no se argumenta que Sam no pueda creer que los habitantes no fueron tragados por los tigres o que el diario no es fiable, con lo cual se encuentra de acuerdo el no reduccionista - por ejemplo, cuando distinguimos entre suposición de cualidad de persona y la presuposición de verdad-; más bien, se afirma que la razón por la cual Sam tiene un derrotero es en base a cuán radical es esta nueva fuente de información y cuán probablemente pueda ser tendenciosa a la falsedad.

Otra segunda objeción que Lackey podría elaborar es argumentando que al admitir la aparición de una contra-creencia normativa, se estaría aceptando que se necesita alguna evidencia inductiva de que la fuente es apta para la producción de verdad. Por tanto, estaría abandonando el no reduccionismo. Pero la respuesta a esta nueva objeción es análoga a la anterior. Sam tiene un derrotero porque cree que el diario fue dejado por un alien, de quien carece evidencia positiva sobre su práctica testimonial, y según su conocimiento de fondo y su experiencia, estos casos no son fiables. Por último, sobre el asunto del intercambio que Lackey hace entre el alien y Sally, una integrante de nuestra institución testimonial, sólo habría que preguntarse cuán seguido sucede el caso expuesto; y esto apunta nuevamente a nuestras creencias y experiencias previas sobre el testimonio.

La razón de que esto sucede, creo - con base en el argumento de Kusch-, es debido a que Lackey hace trabajar en conjunto dos presupuestos con los cuales la crítica se desvía. La suposición de la presuposición de verdad sigue de pie, a menos de que, como en los casos presentes, nos enfrentemos con este tipo de sucesos radicales. 
Por tanto, (1) es falso, y según el reduccionismo, a diferencia de la opinión de Lackey, Sam no se encuentra justificado en creer en el testimonio del alien.

\section{Conclusión}

Hilary Kornblith ha bautizado a diferentes movimientos internistas como "cartesianos impacientes", ${ }^{16}$ por su común ansiedad a considerar que todo conocimiento debe proceder de la reflexión $-o$, mejor dicho, a partir de un proceso metacognitivo-. Elizabeth Fricker es quizá una de las mayores representantes de dicha etiqueta en lo que respecta al testimonio. Su reduccionismo local es el intento de argumentar que la justificación testimonial es producto de nuestras capacidades internas-individuales. Pero las cosas han cambiado, o al menos hemos hallado una nueva metodología que promete nuevas y esperanzadoras soluciones. Me refiero a los movimientos naturalistas, que nos han ofrecido una perspectiva diferente en filosofía. Razón por la cual creo, de manera general, que el reduccionismo $-y$ el internismo- se encuentra en serios problemas. Por ejemplo, en algunos experimentos Nisbet y Lee Ross ${ }^{17}$ muestran que nuestro razonamiento se encuentra influenciado fuertemente por factores independientes de la capacidad reflexiva individual, los cuales, la mayoría de las veces, son inocuos a la introspección. Otro caso es el de Asch, ${ }^{18}$ quien ha señalado que contamos con una tendencia natural a estar influenciados por las opiniones de otros, la cual es inconsciente.

El no reduccionismo parece tener un punto a favor, ya que el reduccionismo junto con la tradición epistemológica, han sufrido incontables críticas. La respuesta ante esto han sido ciertos posicionamientos intermedios, que encontramos principalmente en Jennifer Lackey, quien a su vez ha sido influenciada por Ernesto Sosa y su teoría de las virtudes, la cual intenta conciliar al internismo y al externismo. Mi diagnóstico es que ha quedado una brecha irresoluble entre la tradición y el naturalismo

16 Kornblith, H., op. cit., p. 109.

17 Cfr. Nisbet, R. y Ross, L., Human inference: strategies and shortcomings of social judgement, Nueva Jersey, 1980, p. 207.

18 Cfr. Asch, S., Social Psychology, Prentice Hall, Nueva Jersey, 1952, p. 463-464. Asimismo, para un análisis de dicho experimento y una defensa de esta tendencia natural, véase Kornblith, H., “Some Social Features of Cognition”, Synthese, 73, 1987, pp. $27-41$. 
contemporáneo, a pesar del intento de conciliación. Esto se entiende mejor si nos enfocamos en la pregunta de qué es el conocimiento. Así, Alvin Goldman, ${ }^{19}$ quien de un tiempo acá comparte la idea de una teoría de las virtudes, no obstante con un sesgo marcadamente naturalista y, por ende, empírico, sostiene un tipo de "naturalismo moderado", pues rechaza que el conocimiento sea una clase natural. En lo personal, creo que el conocimiento es una clase natural, ${ }^{20}$ y que la epistemología debe trabajar a la par de la ciencia, más precisamente, de las ciencias cognitivas. Esto implica que debemos eliminar cualquier atisbo de aprioricidad, que se estudie el conocimiento como una característica del mundo, más que como un concepto producto de nuestro entendimiento. Por tanto, si las pruebas empíricas de las ciencias cognitivas - o incluso de estudios antropológicos- nos muestran que en el proceso de justificación testimonial sólo se requiere que no existan derroteros, deberemos cuestionar seriamente al hibridismo y a las posiciones intermedias o naturalistas moderadas en la epistemología.

Concluyo. La respuesta a los contraejemplos de Lackey que hemos abordado en el presente ensayo nos indica que no existe ninguna clase de actitud acrítica en el no reduccionismo. Al contrario, quizá debemos trazar una sutil distinción entre confiar ciegamente y confiar, donde sí existe una oposición. Esto es compatible con estudios antropológicos. Por ejemplo, Dan Sperber et al argumentan que los seres humanos contamos con un conjunto de mecanismos cognitivos para un cierto tipo de "vigilancia epistémica" ${ }^{21}$ Es decir, un oyente $O$ no se encuentra pasivo cuando recibe información de terceros, más bien, podríamos decir que, la mayoría de las veces, es inconsciente del funcionamiento de dichos mecanismos; ambas cosas, por supuesto, son diferentes.

En todo caso, nuestra dependencia en el testimonio es ubicua y necesaria, desde que nacemos y llegamos a interactuar en sociedad, en trabajos colaborativos en ciencia y en los juzgados al tomar una decisión importante. He ahí la razón del enorme estudio que se ha desarrollado en la epistemología social. ${ }^{22}$

19 Así lo hace ver en Goldman, Alvin. "Epistemic Folkways and Scientific Epistemology", en Kornblith, H. (Ed.), Naturalizing Epistemology, 1994, pp. 291-315.

20 Tomo la definición de Richard Boyd como paradigmática. Es decir, una clase natural es un conjunto de "propiedades homeostáticamente agrupadas" (Boyd, R., "How to be a Moral Realism”, en Sayre-McCord, G. (Ed.), Essays on Moral Realism, Cornell University, 1998, pp. 194-199).

21 Sperber, D et al,"Epistemic Vigilance”, Mind and Language, 25, 2010, pp. 359-393.

22 Agradezco a Tahi de León Guzmán, por sus comentarios y correcciones. 


\section{Referencias}

Goldman, A., Knowledge in a Social World, Oxford, 1999.

Lackey, J. y Sosa, E. (Ed.), The Epistemology of Testimony, Oxford, 2006. Burge, T., "Content Preservation", The Philosophical Review, 102.

Lackey, J., Learning from Words: Testimony as a Source of Knowledge, Oxford, 2008.

Kusch, M.,"Jennifer Lackey on Non-Reductionism: A Critique”, 2011. Recuperado de [https://www.academia.edu/1166002/Jennifer_Lackey_on_Non-Reductionism_A_Critique].

Adler, J., "Epistemological Problems of Testimony". Recuperado de [http://plato.stanford.edu/entries/testimony-episprob/].

Perrine, T., "In Defense of Non- Reductionism in the Epistemology of Testimony, Synthese, 191.

Kornblith, H., Knowledge and its Place in Nature, Oxford, 2015.

Kornblith, H., "The Role of Reasons in Epistemology", Episteme, 12.

Nisbet, R. y Ross, L., Human inference: strategies and shortcomings of social judgement, Nueva Jersey, 1980.

Asch, S., Social Psychology, Nueva Jersey, Prentice Hall, 1952.

Kornblith, H., "Some Social Features of Cognition", Synthese, 73.

Goldman, A., "Epistemic Folkways and Scientific Epistemology", en Kornblith, H. (Ed.), Naturalizing Epistemology, 1944.

Boyd, R., "How to be a Moral Realism", en Sayre-McCord, G. (Ed.), Essays on Moral Realism, Cornell University, 1998. 
